\title{
Audrey Guitton, L'Autre lointain en dialogue. La quête de la voix idéale au siècle des Lumières
}

Paris, Classiques Garnier, coll. « L'Europe des Lumières », 2012, 531 p.

\author{
Isabelle Tremblay \\ Collège militaire de Kingston
}

L'Autre lointain en dialogue d'Audrey Guitton fait ressortir avec brio tous les enjeux issus de l'emploi du dialogue pour faire entendre la voix de l'Autre. Il ressort de cet ouvrage que, physiquement ou imaginairement coupé de la société française, l'Autre lointain est instrumentalisé au dix-huitième siècle pour favoriser la connaissance de soi, c'est-à-dire pour se penser 
autrement, pour exprimer ses peurs, ses manques et ses possibles.

La première partie de l'ouvrage passe en revue les différents visages de l'Autre lointain. De l'Anglais à l'Allemand en passant par l'Espagnol, la voix de l'Européen comme «figure-miroir» (p. 44) est familière aux lecteurs français des Lumières et bien qu'elle témoigne de traits distinctifs, elle évite de véhiculer une remise en question de la société française à laquelle elle est étroitement liée. Exclue du cadre de référence, la femme dont la société des Lumières rejette l'altérité est réduite au silence. Audrey Guitton se demande avec raison si la différence au nom de laquelle la femme subit un grand nombre d'injustices contribue malgré tout à représenter la société patriarcale sous un mode différent. Le personnage de l'Africain connaît le même traitement que la femme et sert de repoussoir à l'homme des Lumières. Soustrait aux contraintes qui régissent la société des Lumières, le dialogue des morts fait entendre un discours à la fois personnel et critique. La figure de l'Oriental, qui incarne le contre-modèle négatif de l'Occidental, laisse deviner une "voix relativiste » (p. 64) et engage à la tolérance. Entièrement étranger à la société française, le Sauvage, dont l'innocence fait un juge absolu, esquisse les contours d'un «monde idéal » (p. 101), favorisant par là une « communication transparente» (p. 87). Contrairement au Sauvage, à travers lequel s'exprime la nature, l'Utopien révèle à la société française une vérité sur elle-même grâce à la raison dont il fait preuve. L'auteure fait remarquer que le roman épistolaire, simulacre de transparence et de vraisemblance, est le lieu d'une transformation du scripteur qui, à cause de la distance, n'est plus qui il était, de sorte que la mise en lettres du dialogue entrave la transparence nécessaire pour exprimer pleinement la 
«spécificité de la voix de l'Autre» (p.116). Malgré tout, l'ouvrage en question aurait gagné à traiter davantage des Lettres persanes (1721) de Montesquieu et des Lettres d'une Péruvienne (1747) de Mme de Graffigny, qui jouent un rôle déterminant dans l'émergence de l'altérité à l'époque des Lumières. Audrey Guitton privilégie surtout le théâtre qui, selon elle, constitue un espace idéal où faire entendre la différence de l'Autre lointain à l'aide du dialogue. En se penchant sur l'Arlequin sauvage (1721) de Delisle de la Drevetière, sur La jeune Indienne (1764) de Chamfort et sur Le Huron (1768) de Marmontel, elle convainc aisément le lecteur qu'au théâtre, l'Autre lointain communique aisément son altérité et remplit bien une « fonction réflexive » (p. 137).

L'auteure traite ensuite de la notion de dialogue, dont elle distingue trois types: le dialogue dramatique, qui a tous les traits de la conversation, le dialogue diégétique, qui s'intègre à d'autres procédés narratifs, et le dialogue mimétique, qui s'insère dans un récit de voyage. À l'aide d'exemples tirés de l'Élève de la nature (1771) de Gaspard Guillard de Beaurieu et de Voyages et aventures de Jacques Massé (1710) de Simon Tyssot de Patot, elle montre bien que les dialogues dramatique et diégétique ménagent à l'Autre lointain l'autonomie nécessaire pour affirmer sa différence. Rendu possible par la rencontre de l'inconnu, le dialogue repose sur la découverte d'un espace étranger, qu'il soit imaginaire ou réel, et donne la parole à un Autre qui dit vrai. Bien entendu, le dialogue qui se réclame de la parole recourt malgré tout à l'écriture, filtre susceptible d'altérer la voix idéale. Comment résoudre cette contradiction? 
L'auteure répond à cette question dans la deuxième partie de son ouvrage. À l'exception des Femmes militaires (1739) de Rustaing de Saint-Jory, qui élabore une langue utopienne à base d'ancien français ponctuée d'archaïsmes, la majorité des œuvres mettant en scène la parole de l'Autre recourent à la langue française. La traduction supposée de la langue idéale de l'Autre lointain joue un rôle capital: elle permet de faire connaître sa voix sans pour autant avoir à reproduire sa langue, justifiant ainsi le passage d'un idiome idéal au support imparfait que constitue la langue française. Parce que le langage lèse la communication, comme le déplorait John Locke et ses adeptes au $18^{\text {e }}$ siècle, il conforte l'être humain dans ses préjugés. En s'appuyant sur Zélie ou l'Ingénue (1785) de Mme de Genlis, l'auteure montre que la figure de l'Autre lointain appelé à maîtriser le code de référence remet en question les règles et les codes qui régissent la conversation dans la société française des Lumières. L'étude des Animaux raisonnables (1718) de Fuzelier montre clairement que la parole de l'Autre lointain est chargée d'une fonction « anamorphosique» (p. 257). La logique de renversement mise en œuvre dans l'île de la raison ou les Petits hommes (1727) de Marivaux confirme cette fonction du langage, qui a l'avantage de conférer un pouvoir de réflexion critique à des personnages qui en sont généralement privés.

L'auteure s'interroge ensuite sur l'effet que produit le discours de l'Autre lointain sur l'instance narrative, qu'elle désigne par le terme "énote» (p. 281), c'est-à-dire le lecteur inscrit dans le texte qui incarne le familier et qui reproduit les valeurs de la société de référence. En tant que «figure d'accompagnement du lecteur » (p. 282), il éprouve différentes réactions vis-à-vis du discours que tient l'Autre lointain : rejet, 
adhésion, embarras, rire. La réception du dialogue est déterminée par plusieurs stratégies. Au nombre de celles-ci, on compte ce qu'Audrey Guitton appelle des "phénomènes de dédoublement » (p. 300), tels que la rétrospection, le prologue ainsi que l'alternance de la parole de l'Autre et de celle d'un personnage extérieur à l'action. Dans tous les cas, l'auteure montre que la lecture gagne à être prolongée dans un dialogue qui appartiendrait « pleinement à la société » (p. 311).

La troisième partie explore la nouvelle fonction que remplit la voix de l'Autre lointain dans la seconde moitié des Lumières, soit sa capacité à atteindre l'homme, à le toucher et à « réveiller en lui des sentiments endormis » (p. 318) plutôt qu'à lui imposer un savoir. La voix de l'Autre lointain découvre à l'homme ce qu'il a été amené à étouffer, soit sa voix première, celle que la Nature lui a donnée. Cette "voix intérieure » (p. 321), qui s'oppose à la « voix de la raison » (p. 323), expose les replis de la conscience qui doit régler la conduite des hommes. L'expression de cette voix naturelle procède d'un dialogue intérieur et témoigne de l'émergence de la sensibilité. C'est pourquoi l'ignorance, désormais chargée d'une connotation positive, caractérise à la fois le personnage du Sauvage et celui de l'homme élevé en marge de la société, deux figures incarnant «l'Être de nature» (p. 329). Dans la voix naturelle réside le pouvoir de transformer la société : en ranimant la conscience vertueuse de l'interlocuteur et en lui découvrant une certaine vérité sur lui-même, elle est susceptible de lui faire comprendre l'injustice que perpétue l'ordre établi et de le conduire à l'action. C'est ce qui explique que l'auteure retienne essentiellement des œuvres théâtrales qui, comme elle le soutient, ont l'avantage de "faire naître l'homme sensible » (p. 376). 
Audrey Guitton n'est pas sans souligner qu'un certain nombre d'auteurs, dont Voltaire et Marivaux, témoignent d'une vive opposition face à cette valeur heuristique de la fiction. Alors que les adeptes de Rousseau considèrent l'ignorance et l'innocence dont témoigne l'Autre lointain comme un bien moral, d'autres auteurs y recourent pour faire l'apologie de la civilisation. Dans Frédéric ou l'île inconnue (1765) de FrançoisLouis-Claude Marin, le dialogue occulte la voix de la nature pour ne faire entendre que celle de l'Européen, qu'imite désormais la figure du Sauvage. En devenant une «forme univoque» (p. 396), le dialogue ne se fait plus que l'écho du discours de l'autorité.

Bien que les textes canoniques des Lumières ne s'y taillent qu'une petite place et que l'évolution chronologique esquissée se limite à une division binaire, L'Autre lointain en dialogue a le mérite de faire découvrir un répertoire littéraire peu connu où triomphe le dialogue. Parce qu'il brosse un tableau détaillé de l'élaboration d'un mode de communication capable de faire réfléchir la société de référence sur elle-même, l'ouvrage d'Audrey Guitton jette un éclairage nouveau sur la littérature des Lumières. 